\title{
Identification of Ventricular Tachycardia Using Morphologic Analysis of the Intraventricular Electrogram
}

\author{
By Dongping Lin, Ph.D.,* Lorenzo A. DiCarlo, M.D.,† and Janice M. Jenkins, Ph.D.*
}

Tachycardia detection by implantable antitachycardia devices using rate alone has major limitations. We have examined abnormal ventricular activation in intraventricular electrograms using correlation waveform analysis as a clue to distinguishing ventricular tachycardia from normal sinus rhythm. Thirty episodes of induced, monomorphic ventricular tachycardia were analyzed and compared with sinus rhythm in four groups of patients with (1) normal surface QRS conduction during sinus rhythm without antiarrhythmic drug therapy (5 episodes); (2) intraventricular conduction delay or bundle branch block during sinus rhythm without antiarrhythmic drug therapy (9 episodes); (3) normal surface QRS conduction during sinus rhythm with antiarrhythmic therapy ( 6 episodes); and (4) intraventricular conduction delay or bundle branch block during sinus rhythm with antiarrhythmic drug therapy (10 episodes). Paired data sets, consisting of 10-second passages of both sinus rhythm and ventricular tachycardia in the same patient, were digitized for computer analysis. Each beat was compared with a patient-defined normal template using correlation analysis. A correlation coefficient $\geq 0.87$ yielded a normal (sinus rhythm) classification and those beats that fell below this threshold were classified abnormal (ventricular tachycardia). The correlation of normal beats ranged from 0.874 to 0.999 , and abnormal beats ranged from -0.971 to 0.851 .

Correlation waveform analysis had $100 \%$ sensitivity and specificity in distinguishing ventricular tachycardia from sinus rhythm, even in the presence of intraventricular conduction delay, bundle branch block, and antiarrhythmic drug therapy.

The addition of morphology to rate would provide a more reliable method for discrimination of ventricular tachycardia from sinus rhythm in intracavitary ventricular electrograms. Its computational demands are modest, making it suitable for consideration in an implantable antitachycardia device.

\footnotetext{
* From the School of Engineering and $\dagger$ St. Joseph Mercy Hospital and the School of Medicine, University of Michigan, Ann Arbor, Michigan.

Supported by a grant from the Whitaker Foundation, NSF Grants ECS-8351215 and ECS-8506494, NIH Grant RO1 HL35554, and a grant from the office of Vice President for Research, University of Michigan.

Reprint requests to: Janice M. Jenkins, Ph.D., Department of Electrical Engineering and Computer Science, University of Michigan, Ann Arbor, MI 48109-2122.
} 